\title{
Constraining nuclear PDFs with CMS
}

\author{
Émilien Chapon*† \\ Experimental Physics Department, CERN, CH-1211 Geneva 23, Switzerland \\ E-mail: emilien.chapon@cern.ch
}

Nuclear parton distribution functions are essential to the understanding of proton-lead collisions. We will review several measurements from CMS that are particularly sensitive to nPDFs. W and $\mathrm{Z}$ bosons are medium-blind probes of the initial state of the collisions, and we will present the measurements of their production cross sections in $\mathrm{pPb}$ collisions at $5.02 \mathrm{TeV}$, and as well a asymmetries with an increased sensitivity to nPDFs. We will also report measurements of charmonium production, including the nuclear modification factor of $\mathrm{J} / \psi$ and $\psi(2 \mathrm{~S})$ in $\mathrm{pPb}$ collisions at 5.02 $\mathrm{TeV}$, though other cold nuclear matter effects may also be at play in those processes. At last, we will present measurements of the pseudorapidity of dijets in $\mathrm{pPb}$ collisions at $5.02 \mathrm{TeV}$.

XXV International Workshop on Deep-Inelastic Scattering and Related Subjects

3-7 April 2017

University of Birmingham, UK

\footnotetext{
*Speaker.

${ }^{\dagger}$ On behalf of the CMS Collaboration.
} 


\section{Introduction}

Nuclear parton distribution functions (nuclear PDFs, or nPDFs) are a crucial input to most heavy ion observables in hard probes production. Similarly to their free proton equivalent, they describe the initial state of the collision. They are often defined relative to a free proton PDF reference, $f_{i}^{\mathrm{p} / \mathrm{A}}\left(x, Q^{2}\right)=R_{i}^{\mathrm{A}}\left(x, Q^{2}\right) f_{i}^{\mathrm{p}}\left(x, Q^{2}\right)$. This allows to cancel to first order the dependence on the free proton PDF when considering in nuclear modification factors, $R_{\mathrm{pA}}$ or $R_{\mathrm{AA}}$, that compare proton-nucleus or nucleus-nucleus to a proton-proton reference at the same centre-of-mass energy. Several regions are usually identified in $x$, with different properties of the $R_{i}^{\mathrm{A}}\left(x, Q^{2}\right)$ ratio. At small $x$, below about $10^{-2}$, a suppression of the $\mathrm{nPDF}$ is observed compared to a free proton PDF, and this is the region of shadowing, especially important for quarkonium production at the LHC for instance. At intermediate $x$, up to about $10^{-1}$, the nPDF is enhanced in the anti-shadowing region, a behaviour believed to be related to a compensation for the shadowing in the normalisation of the PDF. At even larger $x$, the nPDF is suppressed again: this is the so-called EMC effect.

The order of magnitude of the modification of the PDFs in a nucleus varies widely with $x$ and $Q^{2}$, but also the model considered, and can reach up to $20-30 \%$. However, nPDFs are currently poorly known, especially in the kinematic range of the LHC, at low $x$ and / or large $Q^{2}$. A lot less data is available to constrain nPDFs, compared to the free proton PDFs. Most of the constraints come from high $x$ and low $Q^{2}$ values, mostly from deep inelastic scattering and Drell-Yan data from fixed target experiments. Pion production from the PHENIX experiment and neutrino data from the CHORUS experiment can also be used. However, the EPPS16 nPDF [1] is currently the only one to include LHC data, from dijet and electroweak boson production. This data allows to test and constrain the nPDFs in a previously uncovered $\left(x, Q^{2}\right)$ range, reaching about $10^{-3}$ in $x$ and $10^{4} \mathrm{GeV}^{2}$ in $Q^{2}$. This data will also soon be included in the other recent nPDF set available, from the nCTEQ group [2].

Nuclear PDFs are better constrained at the $\mathrm{LHC}$ using $\mathrm{pPb}$ data, rather than $\mathrm{PbPb}$ data, for several reasons. First, generally speaking no "hot medium" effects are expected in such a small system, where no quark gluon plasma forms (at least not a long-lived one). No jet quenching is expected either, and such a phenomenon is still unobserved in $\mathrm{pPb}$ collisions. At last, the hard probes measured in $\mathrm{pPb}$ collisions allow a high correlation between their pseudorapidity and the $x_{\mathrm{Pb}}$ of the parton coming from the lead nucleus, the one coming from the proton being described by the free proton PDF. This allows also for a better precision in the measurement of the nPDF effects.

The CMS experiment [3] has performed several measurements with a potential impact on the knowledge of nPDFs. Results from Z [4] and W [5] bosons production bring constraints on the quark and antiquark nPDFs, but suffer from rather low production cross sections (especially for the $\mathrm{Z}$ boson, with a 10 times smaller yield than the $\mathrm{W}$ boson). Dijet production [6] benefits from a much higher production cross section, and mostly constrains the gluon nPDFs. Both electroweak boson and dijet production in $\sqrt{s_{\mathrm{NN}}}=5.02 \mathrm{TeV} \mathrm{pPb}$ data from CMS are sensitive to a large range in $x$, covering the shadowing, anti-shadowing and EMC regions, about $10^{-3}-10^{-1}$, and at $Q^{2}$ scales of the order of $10^{4} \mathrm{GeV}^{2}$. Quarkonia are also very sensitive probes to nPDFs, but they are usually not considered in the nPDF fits, though they would be probing the nPDFs at smaller $x$ and lower $Q^{2}$. This is because the nuclear modification of the PDFs is not the only effect that impacts results in $\mathrm{pPb}$ collisions [7]: other phenomena are likely at play, such as cold nuclear matter energy 
loss, or comover effects. In ultra-peripheral collisions [8], a suppression is observed compared to the expectation without nuclear effects, which is connected to gluon shadowing. However a deeper understanding of the process is needed for the inclusion in nPDF fits, especially beyond leading order.

\section{2. $\mathrm{Z}$ boson in $\mathrm{pPb}$ collisions}
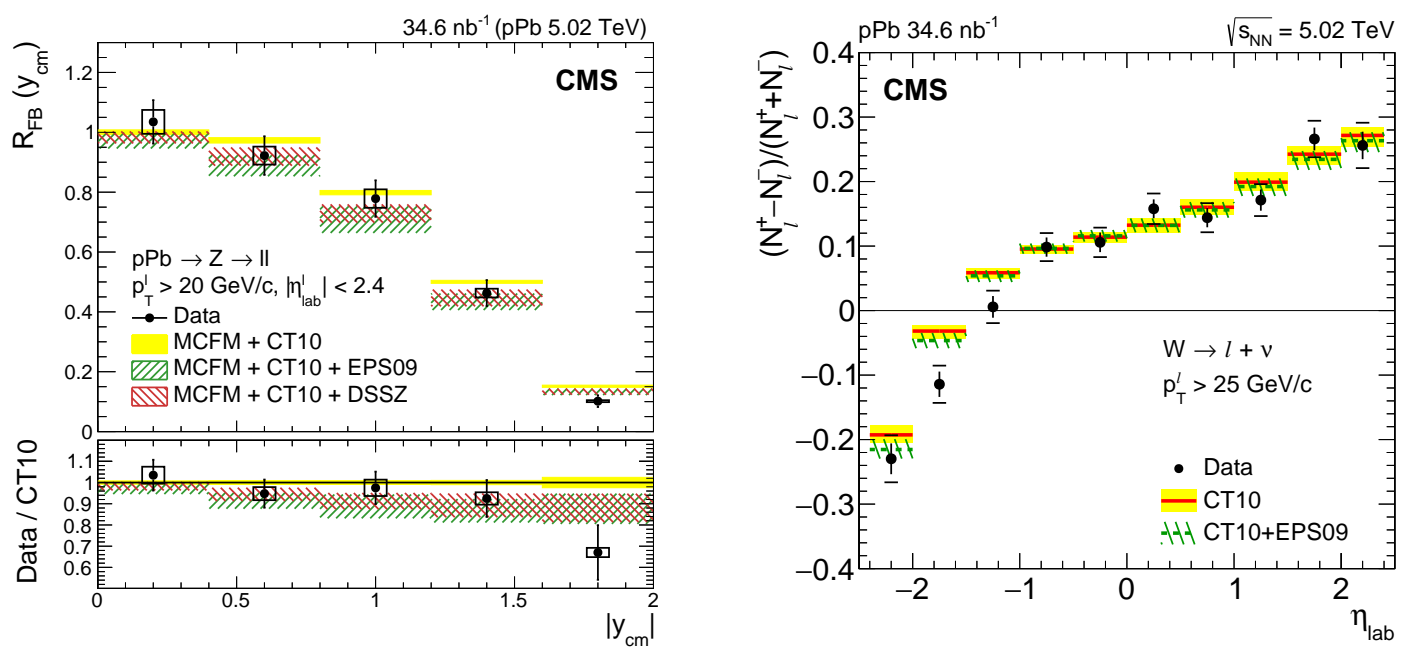

Figure 1: Left: forward-backward asymmetry of the $\mathrm{Z}$ boson production in $\mathrm{pPb}$ collisions [4], as a function of the centre-of-mass rapidity, compared to predictions from MCFM using CT10, CT10+EPS09 or CT10+DSSZ. Right: charge asymmetry in $\mathrm{W}$ boson production in $\mathrm{pPb}$ collisions [5], as a function of the lepton pseudorapidity in the laboratory frame, compared to predictions with and without nPDF modifications from EPS09.

The production of $\mathrm{Z}$ bosons in $\mathrm{pPb}$ collisions at $\sqrt{s_{\mathrm{NN}}}=5.02 \mathrm{TeV}$ has been measured by the CMS experiment in the electron and muon channels [4], using respectively 1571 and 2183 candidates. The measurement has been performed over a large kinematic range, requiring the single leptons to have a transverse momentum $p_{\mathrm{T}}^{\ell}>20 \mathrm{GeV} / \mathrm{c}$ and a pseudorapidity in the laboratory frame $\left|\eta_{\text {lab }}^{\ell}\right|<2.4$.

The fiducial cross section has been measured as a function of the rapidity of the $\mathrm{Z}$ boson in the centre-of-mass frame, combining the results from the two decay channels. These measurements have been compared to several next-to-leading order (NLO) predictions, computed with the MCFM generator [9]. The baseline free proton PDF used is CT10 [10], and computations have been ran without any nPDF modification, or with the modifications given by EPS09 [11] or DSSZ [12]. Uncertainties are large, both on the theoretical and experimental sides, prevent from drawing any firm conclusion at this stage. In order to gain some discriminating power, a forward-backward asymmetry is built from the ratio of cross sections at the same absolute rapidity, in the forward (proton going) and backward (lead going) directions: $R_{F B}=\frac{\frac{d \sigma}{d y}\left(+y_{c . m .}\right)}{\frac{d \sigma}{d y}\left(-y_{c . m .}\right)}$. Several uncertainties cancel in this ratio, both from the experiment and the theory, and the data shows a trend similar to the expectation from EPS09 or DSSZ (see Fig. 1, left). The two models, which differ mainly by their gluon content, cannot be distinguished with the present data. 


\section{3. $\mathrm{W}$ boson in $\mathrm{pPb}$ collisions}

The CMS Collaboration has also measured $\mathrm{W}$ bosons in the same $\mathrm{pPb}$ dataset at $\sqrt{s_{\mathrm{NN}}}=$ $5.02 \mathrm{TeV}$ [5], again using the electron and muon channels. The leptons were required to be with $p_{\mathrm{T}}^{\ell}>25 \mathrm{GeV} / \mathrm{c}$ and $\left|\eta_{\text {lab }}^{\ell}\right|<2.4$. They also have to pass an isolation cut, where the isolation is computed by summing the transverse momenta of the particle flow candidates [13] in a cone around the lepton. The signal extraction is performed using a fit to the missing transverse energy, $\mathbb{E}_{\mathrm{T}}$, without any $\mathbb{E}_{\mathrm{T}}$ cut. The missing transverse energy is reconstructed from the particle flow candidates in the event, it is expected to be higher in $\mathrm{W}$ boson events because of the undetected energetic neutrino. Templates for the signal and the different electroweak backgrounds were obtained from simulation, while the expectation from the multijet QCD background is a parametric shape derived from a sideband of the data with non-isolated leptons.

The production cross section for $\mathrm{W}$ bosons is measured as a function of the lepton pseudorapidity in the laboratory frame, separately for $\mathrm{W}^{+}$and $\mathrm{W}^{-}$. The data has been compared to NLO predictions from CT10 and CT10+EPS09, but large experimental and theoretical uncertainties don't allow for a good discrimination between the two models. However, for the two charges, a $\chi^{2}$ test between the data and the theory, accounting for the correlations, yields to a higher probability when nPDF effects are included, with EPS09.

Asymmetries can be built from this data, which cancel some of the experimental and theoretical uncertainties. The charge asymmetry is defined as $\left(N\left(\mathrm{~W}^{+}\right)-N\left(\mathrm{~W}^{+}\right)\right) /\left(N\left(\mathrm{~W}^{+}\right)+N\left(\mathrm{~W}^{+}\right)\right)$, and is measured as a function as the lepton pseudorapidity in the laboratory frame. If the nuclear modifications to the PDFs are flavour-symmetric, which is assumed to be the case in EPS09 and DSSZ, these modifications cancel in the charge asymmetry. However, some hint for a deviation of the data from the theory prediction is found at large negative lepton $\eta_{\text {lab }}^{\ell}$, at large $x_{\mathrm{Pb}}$, as shown in Fig. 1, right. If confirmed with more data and more precise measurements, such observation could point to a different modification of the up and down quarks in the nucleus. Such an effect is allowed in recent nPDF fits such as nCTEQ15 and EPPS16, though it is poorly constrained at the moment in the available data. A similar tension has also been reported by the ATLAS Collaboration [14]. Interestingly, the charge asymmetry in $\mathrm{pPb}$ collisions can also be connected to that measured in $\mathrm{pp}$ and $\mathrm{PbPb}$ collisions [15], so that more direct comparisons can be made.

As for $\mathrm{Z}$ bosons, forward-backward asymmetries can be built with $\mathrm{W}$ bosons too, for an increased sensitivity to nuclear modifications. The conclusion is unclear when considering each charge separately: while the measurement with negatively charged leptons favours the predictions including nuclear modifications from EPS09, a similar agreement is found between the data from positively charged leptons and the predictions using CT10 or CT10+EPS09. When both lepton charged are combined, the data favours again the presence of nuclear modification of the PDFs, yielding to a $\chi^{2}$ probability of $29 \%$ for CT10, but $83 \%$ for CT10+EPS09.

\section{Dijets in pPb collisions}

Similarly to the case of electroweak bosons, a strong correlation is found between the pseudorapidity of the dijet system and the $x_{\mathrm{Pb}}$ of the parton originating from the lead nucleus. This allows to directly identify the different regions of nPDF modification in a plot of the dijet production as 


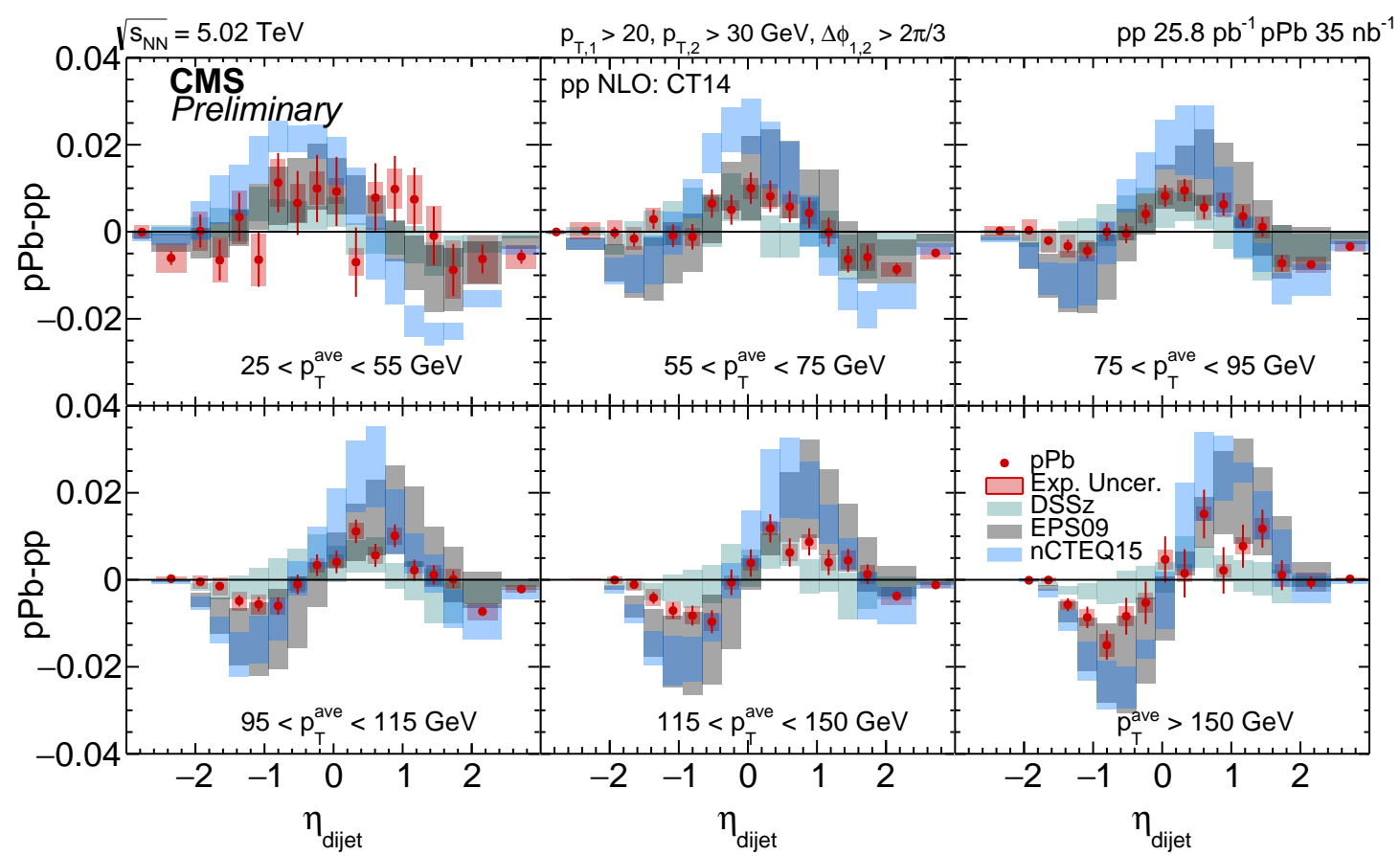

Figure 2: Difference between dijet production in pp and $\mathrm{pPb}$ collisions at $\sqrt{s_{\mathrm{NN}}}=5.02 \mathrm{TeV}$ [6], as a function of the pseudorapidity of the dijet system in the centre-of-mass frame, in several bins of the average $p_{\mathrm{T}}$. The data is compared to predictions from DSSZ, EPS09 and nCTEQ15.

a function of $\eta_{\text {dijet }}$ : large negative pseudorapidities correspond to the EMC effect, midrapidity to antishadowing, and large positive rapidities to shadowing.

The CMS Collaboration has measured dijet production in both $\mathrm{pp}$ and $\mathrm{pPb}$ collisions at $\sqrt{s_{\mathrm{NN}}}=$ 5.02 TeV [6], allowing for a precise and unambiguous investigation of nPDFs, and being less dependent on the fine details of the modelling of dijet production. In particular, the difference between dijet production in $\mathrm{pPb}$ and $\mathrm{pp}$ collisions is reported, as a function of $\eta_{\text {dijet }}$ and in several bins of the average $p_{\mathrm{T}}$ of the two jets, as shown in Fig. 2. The data is compared to NLO predictions from three nPDF models: DSSZ, EPS09 and nCTEQ15. None of these models is able to reproduce the data, in magnitude and shape, simultaneously in all of the measured bins. The experimental uncertainties are smaller than the theoretical ones in most of the bins, especially at intermediate $p_{\mathrm{T}}$, indicating very valuable input to the nPDF fits. Indeed, this data brings significant constraints in the EPPS16 fit, the only one currently including these results.

\section{Quarkonia}

The CMS Collaboration has also reported several measurements of $\mathrm{J} / \psi$ production in heavy ion collisions, which are sensitive to nuclear modifications of the PDFs. The measurement in $\mathrm{pPb}$ collisions at $\sqrt{s_{\mathrm{NN}}}=5.02 \mathrm{TeV}$ [7] is directly probing the nPDFs, in particular with the nuclear modification modification factor $R_{\mathrm{pA}}$, comparing $\mathrm{pp}$ and $\mathrm{pPb}$ data at the same energy and reported as a function of transverse momentum and rapidity. Different models give different expectations, and the size of the experimental uncertainties is often smaller than the theoretical ones. However, 
this data cannot be easily included in nPDF fits, because other effects than nPDFs can be at play. For instance, the modification of $\psi(2 S)$ mesons [16] has been measured to differ from that of $J / \psi$, while a very similar behaviour of the two charmonium states would be expected from nPDF effects, given their close mass.

The measurement of $\mathrm{J} / \psi$ production in ultra-peripheral $\mathrm{PbPb}$ collisions [8] is also sensitive to gluon shadowing, and the data is in good agreement with a model implementing a gluon recombination mechanism. However, the theoretical framework is not ready for the inclusion of this data in next-to-leading order nPDF fits.

\section{Summary}

Nuclear parton distribution functions are a crucial input to the description and understanding of heavy ion collisions. The CMS experiment has performed several measurements, in particular with dijets and electroweak boson production in $\mathrm{pPb}$ collisions, that allow to constrain the nPDFs in a previously uncovered $\left(x, Q^{2}\right)$ space. These measurements start being included in the nPDF global fits, such as EPPS16. Most of the CMS measurements reported in this talk are based on the $\mathrm{pPb}$ data taken at $\sqrt{s_{\mathrm{NN}}}=5.02 \mathrm{TeV}$ in 2013 , but the new data recorded at $\sqrt{s_{\mathrm{NN}}}=8.16 \mathrm{TeV}$ in 2016 will allow much more precise measurements, because of the higher energy and the higher integrated luminosity, by a factor about 5 .

\section{References}

[1] K. J. Eskola, P. Paakkinen, H. Paukkunen and C. A. Salgado, Eur. Phys. J. C 77 (2017) no.3, 163.

[2] K. Kovarik et al., Phys. Rev. D 93 (2016) no.8, 085037.

[3] CMS Collaboration, JINST 3 (2008) S08004.

[4] CMS Collaboration, Phys. Lett. B 759 (2016), 36.

[5] CMS Collaboration, Phys. Lett. B 750 (2016), 565.

[6] CMS Collaboration, CMS-PAS-HIN-16-003 (2016).

[7] CMS Collaboration, Eur. Phys. J. C 77 (2017), 269.

[8] CMS Collaboration, arXiv:1605.06966 [nucl-ex].

[9] J. M. Campbell and R. K. Ellis, Phys. Rev. D 60 (1999) 113006.

[10] H. L. Lai et al., Phys. Rev. D 82 (2010) 074024.

[11] K. J. Eskola, H. Paukkunen and C. A. Salgado, JHEP 0904 (2009) 065.

[12] D. de Florian, R. Sassot, P. Zurita and M. Stratmann, Phys. Rev. D 85 (2012) 074028.

[13] CMS Collaboration, arXiv:1706.04965 [physics.ins-det].

[14] ATLAS Collaboration, ATLAS-CONF-2015-056 (2015).

[15] F. Arleo, É. Chapon and H. Paukkunen, Eur. Phys. J. C 76 (2016) no.4, 214.

[16] CMS Collaboration, CMS-PAS-HIN-16-015 (2017). 